\title{
Coupling of Rayleigh-Wood anomalies and the circular Bragg phenomenon in slanted chiral sculptured thin films
}

\author{
F. Wang ${ }^{\mathrm{a}}$, A. Lakhtakia ${ }^{\mathrm{b}}$, and R. Messier ${ }^{\mathrm{c}}$
}

CATMAS - Computational and Theoretical Materials Sciences Group, Department of Engineering Science and Mechanics, Pennsylvania State University, University Park, PA 16802-6812, USA

The square matrixes $\left[\underline{\underline{Y}}_{\mathrm{e}}\right]^{*}$ and $\left[\underline{\underline{Y}}_{\mathrm{h}}\right]^{*}$ in equation (43) of the subject paper [1] should correctly read as

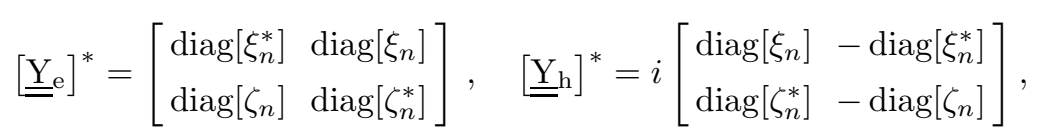

where $\operatorname{diag}\left[\xi_{n}\right]$ is a diagonal matrix containing $\xi_{n}$, etc., and

$$
\begin{aligned}
\xi_{n}^{*} & =\frac{1}{\sqrt{2}}\left(\frac{k_{x n} k_{z n}}{k_{0} k_{x y n}}+i \frac{k_{y 0}}{k_{x y n}}\right), \\
\zeta_{n}^{*} & =\frac{1}{\sqrt{2}}\left(\frac{k_{y 0} k_{z n}}{k_{0} k_{x y n}}+i \frac{k_{x n}}{k_{x y n}}\right) .
\end{aligned}
$$

The presented numerical results are not affected. Any inconvenience is regretted.

\footnotetext{
${ }^{\text {a }}$ e-mail: fuw101@psu.edu

b e-mail: axl4@psu.edu

c e-mail: mes17@psu.edu
} 